\title{
Control Strategy and Implementation for a Humanoid Robot Pushing a Heavy Load on a Rolling Cart
}

\author{
Louis Hawley and Wael Suleiman
}

\begin{abstract}
In this paper, we introduce a control strategy aimed at generating a stable walking pattern for a humanoid pushing a heavy load on a cart. In contrast to previous approaches that rely on force/torque sensors to measure the interaction between the robot and the pushed object, we present a simple model-based controller that can be implemented on most robots due to its computationally efficient design. Every aspect of the controller design is covered, from the formulation and validation of the dynamic model, to the implementation and validation on an actual humanoid robot. The experimental results show that the controller can efficiently make a NAO humanoid transport, in a stable way, the equivalent of its own weight on a rolling cart.
\end{abstract}

\section{INTRODUCTION}

In order to work as efficiently as possible, robots should be able to make use of their environment or available tools. In the case of humanoid robots, one of their biggest strength is their high mobility due to the biped structure. However, it is also a weakness as they are usually unable to carry heavy load without being destabilized contrarily to a wheeled-robot that remains stable. A potential solution to this problem is simply using mobile carts to transport massive or heavy object. However, in order to use such device efficiently, the humanoid robots navigation schemes and control algorithms should be accordingly modified to take the cart into consideration.

In our previous work [1], we presented a navigation approach for a humanoid robot pushing a heavy object on a rolling cart in a cluttered environment. This approach could effectively be used to transport a load that the humanoid robot would not have been able to carry otherwise. However, the dynamic of the system was not analyzed and no corrections were applied on the walking gait of the robot to compensate for the added mass transported by the robot. The focus of this research is to analyze the impact of the cart and transported load on the humanoid stability and come up with appropriate and computationally efficient solutions to control the whole system.

\section{A. State of the art}

A controller architecture for pushing an object by humanoids is presented in [2]. In that work, the authors analyzed the dynamic of a humanoid pushing an object with the arms and assessed that the desired Zero Moment Point (ZMP) trajectory must be modified to keep the robot stable

Louis Hawley and Wael Suleiman are with Electrical and Computer Engineering Department, the Faculty of Engineering, University of Sherbrooke, Sherbrooke, Canada \{louis.hawley, wael.suleiman\}@usherbrooke.ca

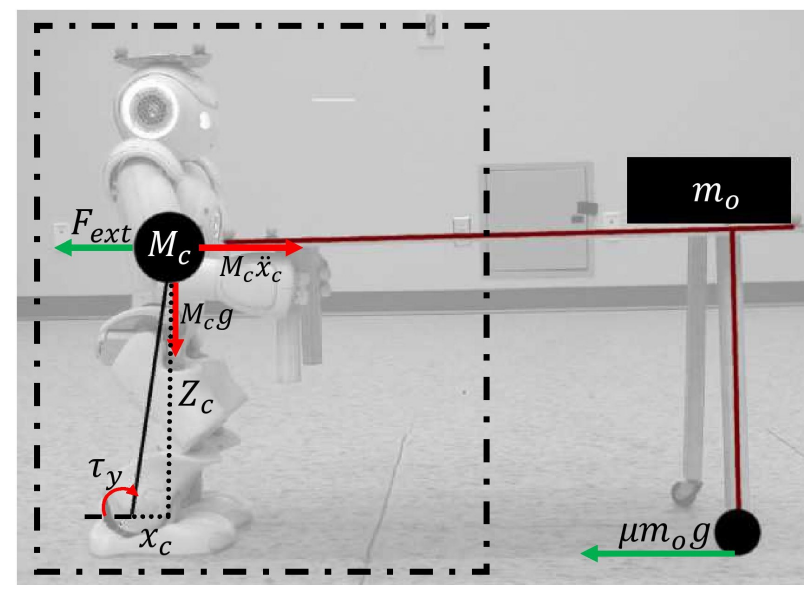

Fig. 1. Humanoid robot pushing a load on a rolling cart

despite the external force generated by the object weight and the friction with the floor. The required ZMP shift is defined as a function of the external force detected by the robot. Thus, the dynamic behaviour of the pushed object is not considered as it is only approximated as a constant external force. Moreover, the authors do not point out how the external force, resulting from pushing the object, should be measured or approximated.

In a more recent work [3], the same authors implemented a controller on a HRP-2 robot pushing a table. The proposed approach is to only push the object while the robot is in a double-support phase. While in double-support phase, a force-controlled loop is driving the arm of the robot in order to apply a desired force on the object. Also, the reference ZMP position is modified to ensure that the robot remains stable considering the reference force applied by the arms on the object. Although this approach is efficient for pushing a heavy object on the floor, a significant drawback is that the force-controlled loop requires accurate measurements of the force applied by the robot's hands. Those forces are measured using force/torque sensors in the wrists of the humanoid robot and these sensors are not available on all robot, e.g. medium-sized humanoid robots such as Nao or Darwin-OP.

Using wheeled platforms to help humanoids transport objects has also received some attention in the literature as it is a skill that could be useful in both a manufacturing or personal robotics context. For example, in [4], a HRP2 robot is used to push a variety of objects ranging from a wheelchair to a simple holonomic transport cart. The authors 
assessed the importance of integrating the force generated by the pushed object in the walk controller to ensure stability. The proposed system is based on an online friction estimator [5] that is able to estimate the friction forces generated by the object using measurements from force/torque sensor located in the wrists and ankles of the robot. Then, the desired ZMP trajectory is modified and supplied to the preview controller to consider the external force. Again, a disadvantage of this approach is that it relies heavily on the good performance of the force/torque sensors. Moreover, according to their approach, the robot must be controlled in force in order to estimate the friction force. However, a lot of humanoid robots are only position-controlled and do not possess such sensors, it would be therefore interesting to have another method that does not rely too heavily on the measurement of those interaction forces.

In most research related to pushing objects by humanoid robots, modelling the external forces is not really treated as those forces can be reliably and directly measured with the available sensors. However, there are multiples advantages of having a model of the force generated by the object as this knowledge can be useful to predict how that force will vary in different conditions.

\section{B. Contribution}

In this work, we present a different control strategy for a humanoid robot pushing a heavy weight on a mobile-cart. The proposed method enhances the simple Linear-Inverted Pendulum Model (LIPM) of a humanoid robot to also include the dynamic model of the cart-load system. Contrarily to the other previously presented methods, our procedure can be used by position-controlled robots that are not equipped with expensive force/torque sensors. In addition, our approach could predict the external force generated by the rolling-cart system as the environment changes (ex: change in floor type or presence of a slope) as illustrate in Fig. 2.

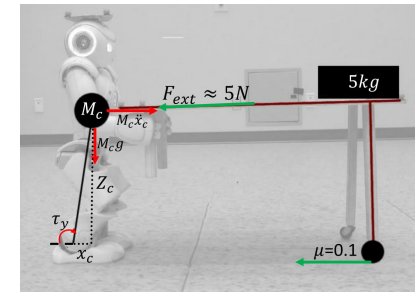

(a) Flat

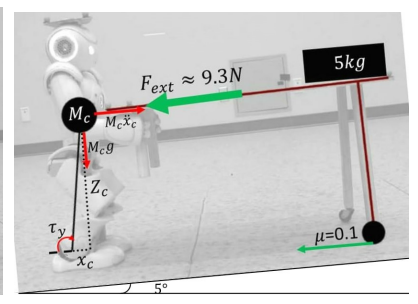

(b) $5^{\circ}$ slope
Fig. 2. The external force generated by the cart and mass on a flat floor and on a small slope. In this case, the force exerted on the robot is nearly doubled in the case of slope compared to a flat floor. A model of the external force can be used to predict such variation and give an idea if the robot can push the cart up the slope or if it should find another path.

The article is organized as follows. The dynamic model of the cart-pushing task and the identification of the cart parameters are presented in Section II. Section III tackles the problem of maintaining the robot stability while executing the transportation task with the cart. Finally, the results of our experiments carried out on a Nao humanoid are presented in section IV.

\section{DYNAMIC MODELS}

\section{A. LIPM dynamic with an external force}

In this work, the LIPM is used to generate a stable walking pattern [6]. In the case of an external force in the horizontal plane, $F_{e x t}=\left[F_{x} F_{y} 0\right]^{T}$, the motion dynamic can be written as follows:

$$
\begin{aligned}
& M_{c} \ddot{x}_{c}=\frac{M_{c} g}{Z_{c}} x_{c}+\frac{1}{Z_{c}} \tau_{y}+F_{x} \\
& M_{c} \ddot{y}_{c}=\frac{M_{c} g}{Z_{c}} y_{c}-\frac{1}{Z_{c}} \tau_{x}+F_{y}
\end{aligned}
$$

where $M_{c}$ is the mass of inverted pendulum, $Z_{c}$ is the height of CoM, $g$ is the magnitude of gravity acceleration, $x_{c}, \ddot{x}_{c}, y_{c}$ and $\ddot{y}_{c}$ and are respectively the position and the acceleration of the projection of CoM on the $x$ and $y$ axis. $\tau_{x}$ and $\tau_{y}$ are respectively the torques around $x$ and $y$ axis.

In a previous work [7], we proposed a method to estimate an external force applied in the horizontal plane. This method is mainly designed for small humanoid robots, such as Nao robot, and only uses the information from Force Sensitive Resistors (FSR) and Inertial Measurement Unit (IMU).

\section{B. Cart-pushing task dynamic}

The dynamic model of the cart-pushing task presented in Fig. 1, in which the robot is moving forward in the sagittal plane ( $x$-axis), is defined as follows:

$$
\begin{aligned}
M_{c} \ddot{x}_{c} & =\frac{M_{c} g}{Z_{c}} x_{c}+\frac{1}{Z_{c}} \tau_{y}-F_{\text {ext }} \\
M_{c} \ddot{y}_{c} & =\frac{M_{c} g}{Z_{c}} y_{c}-\frac{1}{Z_{c}} \tau_{x} \\
F_{\text {ext }} & =m_{o} \ddot{x}_{c}+\mu m_{o} g
\end{aligned}
$$

where $\mu$ is the coefficient of rolling friction and $m_{o}$ is the mass of the load on the cart. We suppose that the mass is placed above the cart legs.

From the previous equations, one can see that the origin of the external force can be decoupled into two terms, the first term being the result of the added mass and the second one by the rolling friction on the wheels. The rolling friction coefficient is a function of multiple parameters that are intrinsic to the cart, mainly the wheels diameter and hardness, and also to some other external parameters such as the floor type and surface condition. From Eq.(2), provide that the rolling friction coefficient is known, the mass carried on the cart can be easily estimated by measuring the external force and the acceleration as follows:

$$
m_{o}=\frac{F_{e x t}}{\mu g+\ddot{x}_{c}}
$$

The following section provides a simple way to estimate the rolling friction coefficient of a cart.

\section{Cart Parameter Identification}

In order to generate a stable walking pattern while pushing the cart, it is possible to measure the force generated by the cart and then directly incorporate this force in the stability 


$-\mu=0.06-\mu=0.07-\mu=0.08-\mu=0.09--$ Actual mass

Estimated mass

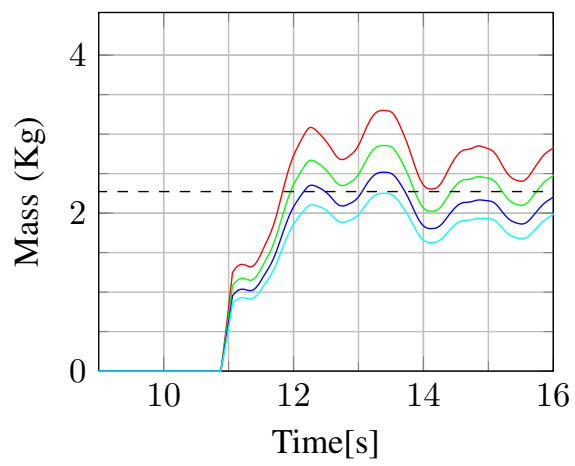

(a) Mass of $2.3 \mathrm{Kg}$
Estimated mass

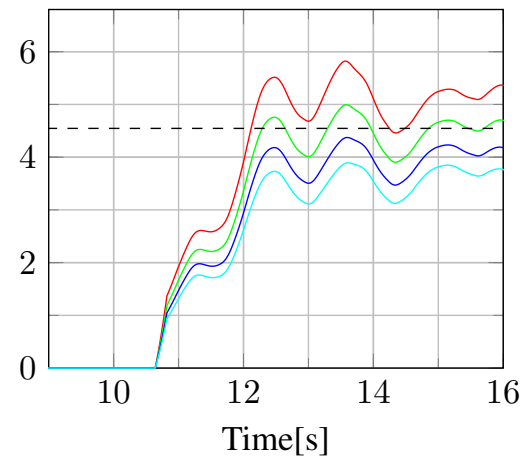

(b) Mass of $4.5 \mathrm{Kg}$
Estimated mass

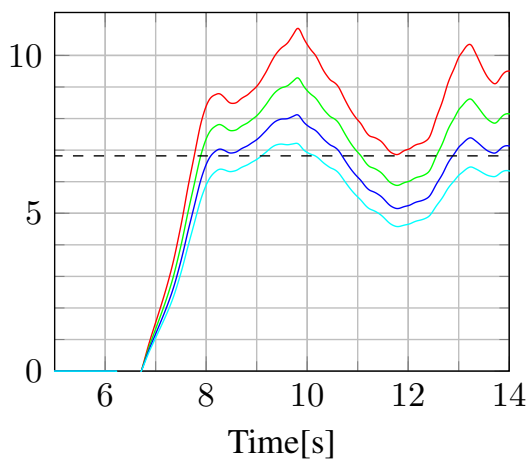

(c) Mass of $6.8 \mathrm{Kg}$

Fig. 3. Estimation of the transported mass for three experiments using Eq. (3) and varying rolling friction coefficient $\mu$

control loop of the robot. However, as above-mentioned, having an estimation of the transported load can sometimes be really important in the motion planning phase. For example, while a heavy load can be relatively easy to transport with a cart on a hard and flat floor, it can be much more difficult if the robot pushes the cart on a carpet or on a slope. Such knowledge can be used by the path planner to prevent from accessing zones that will likely destabilize the robot or to optimize the energy consumption of the robot by avoiding high-friction surfaces or steep slopes.

A simple way to measure the friction coefficient of an object is presented in [8], where the authors slowly increase the force applied by the humanoid robot arms on the object. At some point, the object starts to move, and thus the measured force stabilizes at a given value. If the mass of the object $m_{o}$ is known, the friction coefficient $\mu$ can then easily be found with $F=\mu m_{o} g$. However, it is not possible to reproduce this procedure with a Nao robot since the motor can only be controlled in position.

In order to estimate the parameters of the cart, three experiments were performed with a Nao robot pushing different masses using a rudimentary wheeled-table. In each experiment, we estimated the external force applied on the robot using our force observer [7], and tried to correctly guess the mass transported on the cart by using Eq. (3) and varying the cart-parameter $\mu$.

The results of those experiments along with the mass estimation using different $\mu$ values are presented in Fig. 3 . Note that in this case, the parameter $\mu$ combines both the intrinsic cart-parameter, i.e. wheel diameter, and environmental parameters, i.e. floor type, since our laboratory has a uniform floor. Otherwise, the experiments would have been conducted on the different available surfaces and the coefficient would have been split into two terms. A quick look at the results shows that a $\mu$ value of about 0.07 yields a reasonable estimate for the three masses, and this value is therefore used in the sequel of the paper. The following section takes a look at the effect of the carried mass on the robot ZMP in simulation as well as on a real Nao robot.

\section{Model Validation}

Using the dynamic model presented in section II-B, the ZMP dynamic was simulated in Matlab for a scenario where a humanoid robot pushes the previously characterized cart with a mass of $5 \mathrm{~kg}$. Recalling that the ZMP is the point on the floor where the sum of the moment is null, it is expressed as :

$$
\begin{aligned}
& x_{Z M P}=x_{c}-\frac{Z_{c}}{g} \ddot{x}_{c}-\frac{Z_{c}}{M_{c} g} F_{e x t} \\
& x_{Z M P}=x_{c}-\frac{Z_{c}}{g} \ddot{x}_{c}-\frac{m_{o} Z_{c}}{M_{c} g} \ddot{x}_{c}-\frac{\mu m_{o} Z_{c}}{M_{c}} \\
& x_{Z M P}=x_{c}-\frac{\left(m_{o}+M_{c}\right) Z_{c}}{M_{c} g} \ddot{x}_{c}-\frac{\mu m_{o} Z_{c}}{M_{c}} \\
& y_{Z M P}=y_{c}-\frac{Z_{c}}{g} \ddot{y}_{c}
\end{aligned}
$$

Where $x_{Z M P}$ and $y_{Z M P}$ are the projections of the ZMP on the floor $(x-y$ plane).

Fig. 4(a) and Fig. 4(b) respectively presents the planned and actual global ZMP in the sagittal $\left(x_{Z M P}\right)$ and coronal $\left(y_{Z M P}\right)$ plane of the robot as the mass is added on the cart at $T \approx 4 s$. The local $x_{Z M P}$ (expressed in the support foot ankle frame) is also presented in Fig. 4(c) to clearly show the effect of the cart and mass on the ZMP dynamic. First, the rolling friction caused by the cart wheels generates a constant shift of the ZMP toward the back. Second, the mass carried on the cart generates a dynamic force as a result of the robot center of mass acceleration. Fig. 4(b) validates that in the case of a forward push, the $y_{Z M P}$ is not affected.

The experiment was then carried out on a real Nao robot to validate the simulation results. Fig. 5 presents the local ZMP position during the conducted experiment. At first the NAO humanoid is pushing an empty cart. Then, at $T \approx 22 s$, a mass of $5 \mathrm{~kg}$ was added on the cart. Fig. 5 points out that the ZMP shift is similar to what was found in simulation 


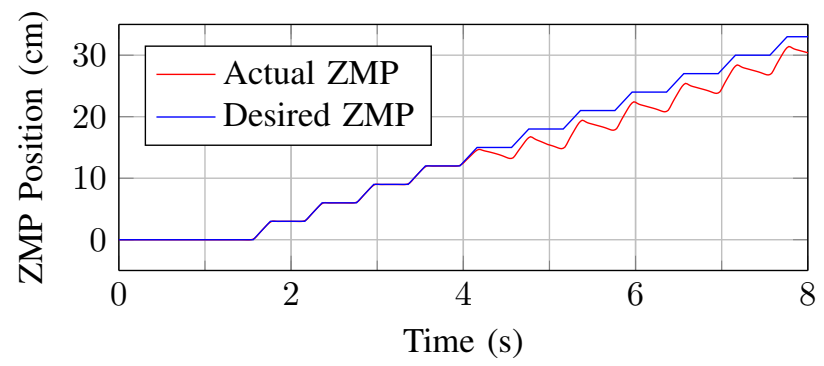

(a) Global ZMP in the sagittal plane (x-axis)

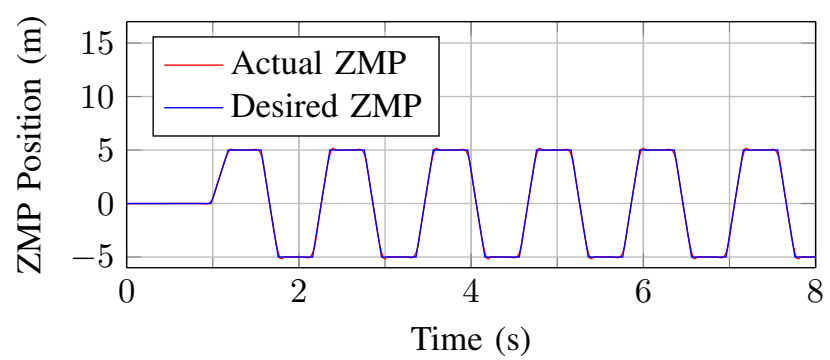

(b) Global ZMP in the coronal plane (y-axis)

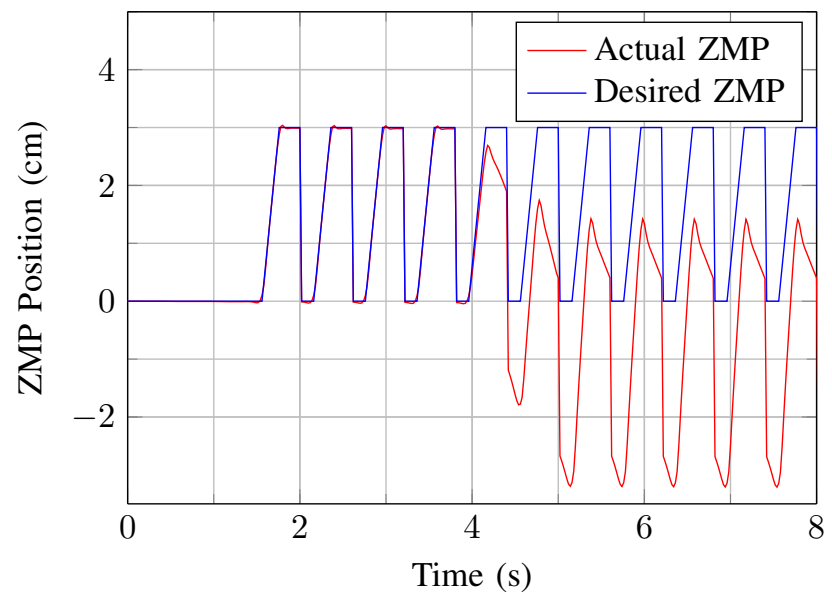

(c) Local ZMP in the sagittal plane (x-axis)

Fig. 4. Simulation result for a scenario where a mass of $5 \mathrm{~kg}$ is added on a cart pushed by a Nao humanoid. The ZMP in the coronal plane (b) of the robot is not affected in this case contrarily to the ZMP in the sagittal plane((a) and (c)).

as the ZMP moves approximately $2 \mathrm{~cm}$ backward whereas a shift of approximately $2.4 \mathrm{~cm}$ was found in simulation.

\section{CONTROL LAW}

\section{A. ZMP Preview Control}

Now that we have a valid model for the external force generated by the rolling-cart and the transported mass, a control law considering the whole dynamic must be developed.

To this end, we designed an optimal preview servo controller following the method defined in Kajita et al [9].

Let us first define a new variable $p$ such that :

$$
p(t)=\left[\begin{array}{c}
x_{Z M P}+\frac{\mu m_{o} Z_{c}}{M_{c}} \\
y_{Z M P}
\end{array}\right]
$$

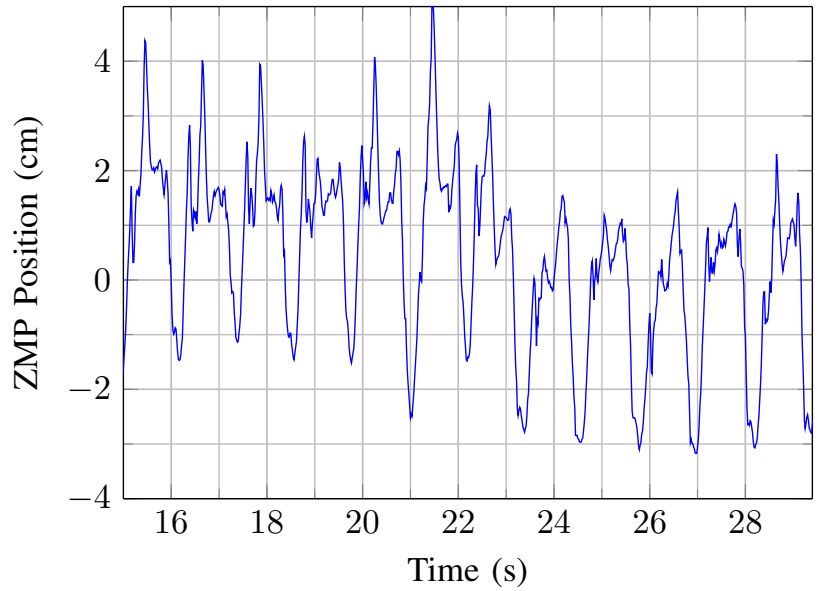

Fig. 5. The local ZMP measured during an experiment. At around $T=$ $22 \mathrm{~s}$, a mass of $4 \mathrm{Kg}$ was added on the cart

The ZMP equations in (4) are then discretized at a sampling period $T$, and expressed as a Linear Time Invariant (LTI) system as follows:

$$
\begin{aligned}
\mathcal{X}(k+1) & =A \mathcal{X}(k)+B u(k) \\
p(k+1) & =C \mathcal{X}(k)
\end{aligned}
$$

where

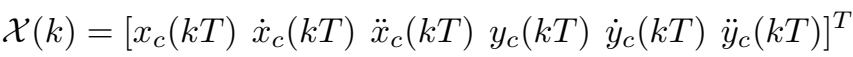

$$
\begin{aligned}
& u(k)=u(k T)=\left[\dddot{x}_{c}(k T) \quad \dddot{y}_{c}(k T)\right]^{T} \\
& p(k)=p(k T) \text {, } \\
& A=\left[\begin{array}{cccccc}
1 & T & \frac{T^{2}}{2} & 0 & 0 & 0 \\
0 & 1 & T & 0 & 0 & 0 \\
0 & 0 & 1 & 0 & 0 & 0 \\
0 & 0 & 0 & 1 & T & \frac{T^{2}}{2} \\
0 & 0 & 0 & 0 & 1 & T \\
0 & 0 & 0 & 0 & 0 & 1
\end{array}\right], \quad B=\left[\begin{array}{cc}
\frac{T^{3}}{6} & 0 \\
\frac{T^{2}}{2} & 0 \\
T & 0 \\
0 & \frac{T^{3}}{6} \\
0 & \frac{T^{2}}{2} \\
0 & T
\end{array}\right] \\
& C=\left[\begin{array}{cccccc}
1 & 0 & -\frac{\left(m_{o}+M_{c}\right) Z_{c}}{M_{c} g} & 0 & 0 & 0 \\
0 & 0 & 0 & 1 & 0 & -\frac{Z_{c}}{g}
\end{array}\right]
\end{aligned}
$$

We can then find the optimal feedback gain to minimize the following cost function:

$$
J=\sum_{i=k}^{\infty}\left\{Q_{e} e(i)^{2}+\Delta x^{T}(i) Q_{x} \Delta x(i)+R \Delta u^{2}(i)\right\}
$$

where $e(i)$ is the error between the reference and the actual ZMP defined as follows:

$$
\begin{aligned}
e(k) & =p^{r e f}(k T)-p(k T) \\
& =\left[\begin{array}{c}
x_{Z M P}^{r e f}(k T)+\frac{\mu m_{o} Z_{c}}{M_{c}} \\
y_{Z M P}^{r e f}(k T)
\end{array}\right]-p(k T)
\end{aligned}
$$

where $x_{Z M P}^{r e f}$ and $y_{Z M P}^{r e f}$ are the ZMP reference trajectories. $Q_{x}, Q_{e}$ and $R$ are, respectively, the weights applied on the 
state vector, on the ZMP error and on the input. The control law that minimizes the cost function $J$ for $\mathrm{N}$ previewed steps is defined as :

$u(k)=-G_{i} \sum_{i=0}^{k} e(k)-G_{x} x(k)-\sum_{j=1}^{N_{L}} G_{p}(j T) p^{r e f}((k+j) T)$

where $G_{i} \in \mathbb{R}^{2 \times 2}, G_{x} \in \mathbb{R}^{2 \times 6}$ and $G_{p}(j T) \in \mathbb{R}^{2 \times 2}$ are the optimal gains.

This control law can take care of the two ZMP perturbation sources:

1) The first source is the constant shift caused by the cart friction, and is compensated by adding an offset of $\frac{\mu m_{o} Z_{c}}{M_{c}}$ to the reference trajectory of ZMP.

2) The second one is related to the transported mass inertia, and is compensated by applying the appropriate optimal gain matrices $G_{i}, G_{x}$ and $G_{p}$.
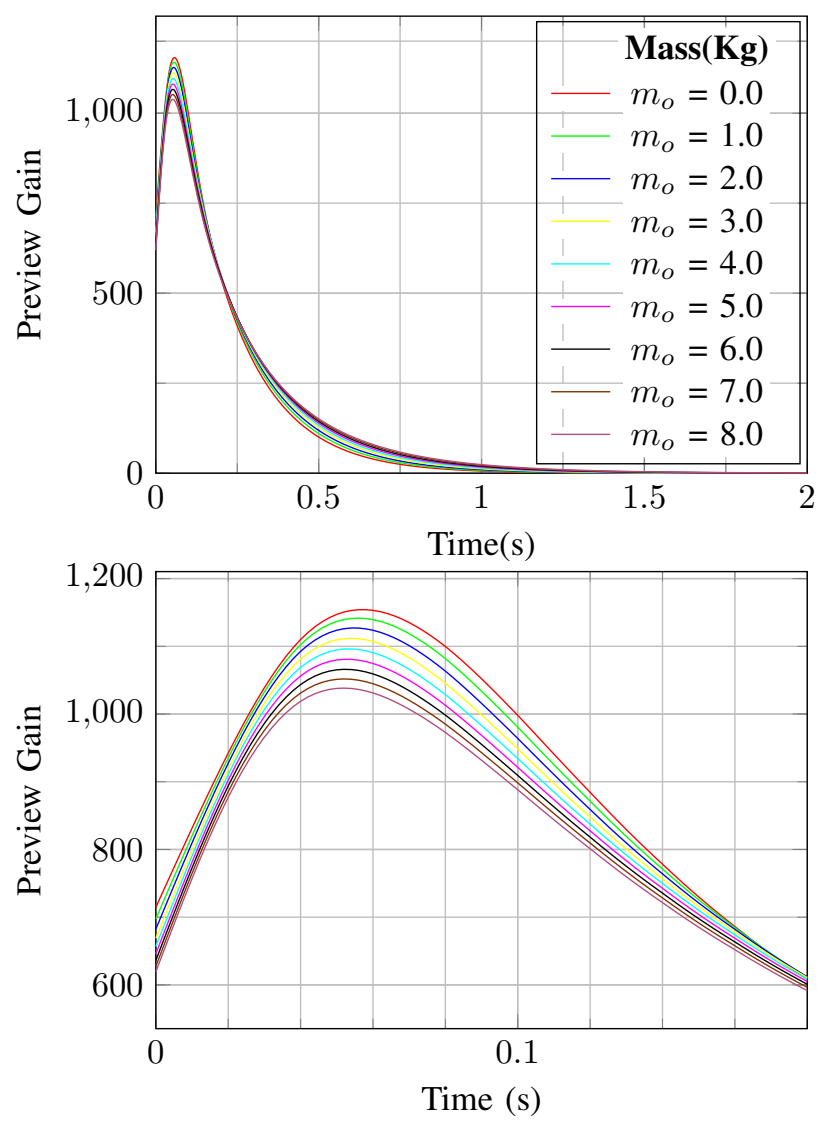

Fig. 6. The norm of each matrix in the gains vector $G_{p}$ for different mass and a close-up of the gain

\section{B. Feedback Gain Analysis}

The feedback gains $\left(G_{i}, G_{x}\right.$ and $\left.G_{p}\right)$ are usually computed offline since their values only depend on the matrices $\{A, B, C\}$ of the LTI system. However, in our case, the transported mass $m_{O}$ appears in the $\mathrm{C}$ matrix, and thus those gains would need to be obtained each time a new mass is detected. Although it is possible to recompute the gains online, by solving Riccati equation, it adds a computational overhead that may be problematic since a gait controller must comply to strict real-time constraints.

As a first step, the preview gains were analyzed for a transported mass ranging between 0 and $8 \mathrm{~kg}$, the latter being the maximum weight the cart can carry before compromising its structural integrity. Interval of $1 \mathrm{~kg}$ was chosen because our external force observer can only reliably detect a force within a $20 \%$ error margin and a $20 \%$ error on $4 \mathrm{Kg}$, which is the mean of our usable weight range, is close to $1 \mathrm{Kg}$. Fig. 6 presents the norm of the different matrices $G_{p}$ in the preview gain according to the transported mass $m_{o}$. It is worth to point out that:

- The gains become close to zero at around $1.5 \mathrm{~s}$ independently from the transported mass $m_{o}$.

- A difference of $1 \mathrm{~kg}$ does not seem to significantly change the gains.

Therefore, instead or recomputing the optimal gains, the controller will simply choose the more appropriate gain from this offline pre-computed set when a new mass is detected on the cart.

\section{Controller Validation}

The controller was implemented and validated in Matlab using the model (4) presented in Section II-D. Fig. 7 presents the local ZMP variation when a humanoid pushes a mass of $5 \mathrm{Kg}$ on a cart: (a) only the cart friction is compensated, (b) only the mass inertia is compensated and (c) full compensation of the external perturbation. As can be seen, the perturbation due to the mass inertia is resolved faster than that one due to the friction. This is because the optimal gains can be changed at anytime while the friction compensation is only done by modifying the reference ZMP at the end of the preview window, which is $1.5 \mathrm{~s}$ in this case.

\section{EXPERIMENTAL RESULT}

Considering the good performance of the controller in simulation, it was implemented on Nao robot.

The controller validation is carried out using the following scenario:

- At the beginning, the robot walks in a straight path pushing an empty cart.

- A mass of $5 \mathrm{~kg}$ is then added on the cart at $T=T_{0}$ while the robot continues to walk as usual.

- The robot estimates the mean of detected external forces over an interval of 4 steps (about 3 seconds) [7].

- At $T=T_{0}+5 \mathrm{~s}$, the robot controller is triggered manually and the transported mass is estimated using Eq.(3), and then integrated into the walk pattern generator using the control law presented in Section III.

Fig. 8 presents local ZMP measurements during the experiment. As can be seen, the controller is able to move the ZMP back towards its initial position; thus can effectively be used to generate a more stable walking gait for a humanoid robot transporting a load with a rolling cart. 


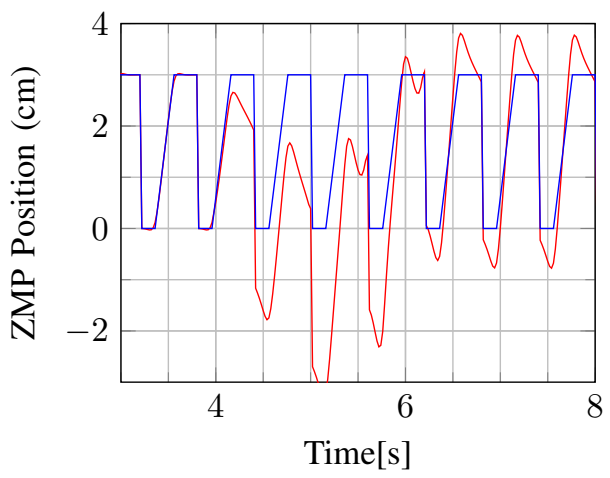

(a) Friction compensation only

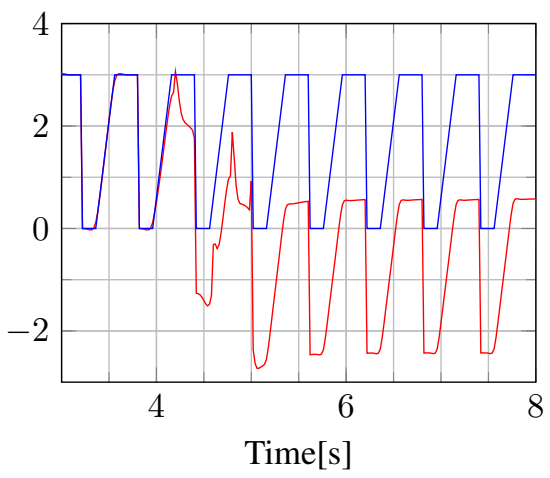

(b) Mass inertia compensation only

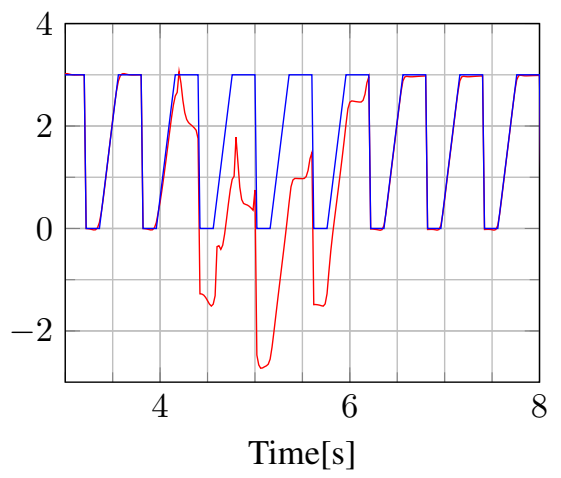

(c) Full Compensation

Fig. 7. Simulation: position of the local ZMP in the sagittal plane of a humanoid pushing a cart. At approximately $T=4 s$, a mass of $5 \mathrm{~kg}$ is added on the cart. The controller then modifies the walking gait to take into consideration the cart friction, the transported mass or both. As a result, the ZMP moves back to its natural position.

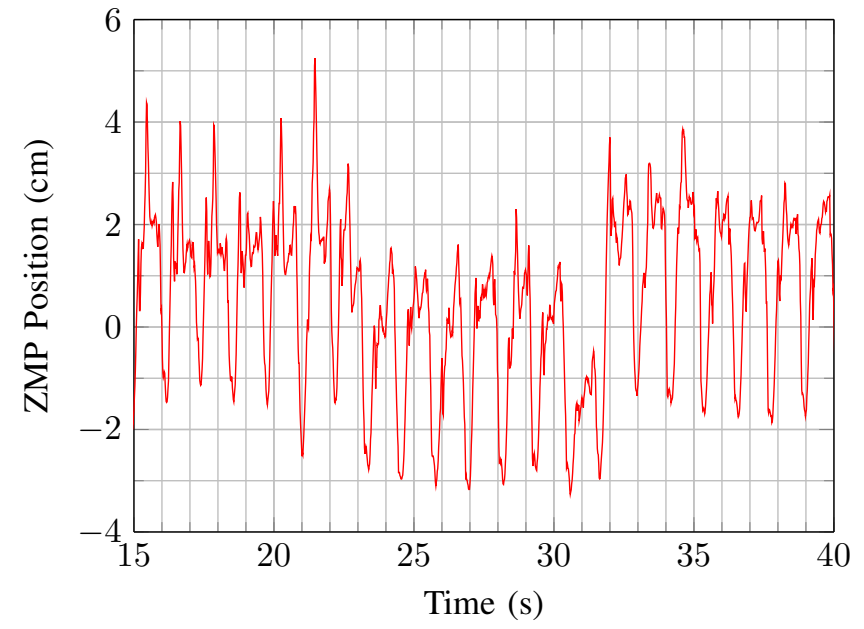

Fig. 8. Local ZMP measurements during a cart-pushing experiment. At $T \approx 22 s$, an unknown mass is added on the cart. At $T \approx 29 \mathrm{~s}$, the controller modified the humanoid walking gait to take into consideration the cart friction and transported mass. As a result, the ZMP moves back to its natural position (regular walk) at $T \approx 32 s$.

\section{CONCLUSION}

In this paper, we introduced a model-based controller for a humanoid pushing a heavy mass on a cart. As we demonstrated in simulation and experiments, the controller enables a humanoid to push a rolling cart more stably by effectively repositioning the ZMP towards the middle of the feet. Also, due to its low hardware (sensor) and computational requirements, the controller can be implemented on

Although path planning was not the focus of this work, the dynamic models developed in this paper could also be used in determining a feasible and optimal path while considering the limitations of the humanoid robot, the characteristics of the cart and the transported load. most humanoid robots.

\section{ACKNOWLEDGMENT}

This research is supported by the Natural Sciences and Engineering Research Council of Canada (NSERC).

\section{REFERENCES}

[1] A. Rioux and W. Suleiman, "Humanoid Navigation and Heavy Load Transportation in a Cluttered Environment," in 2015 IEEE International Conference on Intelligent Robots and Systems (IROS), 2015, pp. 21802186.

[2] K. Harada, S. Kajita, K. Kaneko, and H. Hirukawa, "Pushing manipulation by humanoid considering two-kinds of ZMPs," 2003 IEEE International Conference on Robotics and Automation (Cat. No.03CH37422), no. 1, pp. 1627-1632, 2003.

[3] K. Harada, S. Kajita, F. Kanehiro, K. Fujiwara, K. Kaneko, K. Yokoi, and $\mathrm{H}$. Hirukawa, "Real-time planning of humanoid robot's gait for force-controlled manipulation," IEEE/ASME Transactions on Mechatronics, vol. 12, no. 1, pp. 53-62, 2007.

[4] S. Nozawa, Y. Kakiuchi, K. Okada, and M. Inaba, "Controlling the planar motion of a heavy object by pushing with a humanoid robot using dual-arm force control," Proceedings - IEEE International Conference on Robotics and Automation, pp. 1428-1435, 2012.

[5] S. Nozawa, M. Ishida, R. Ueda, Y. Kakiuchi, K. Okada, and M. Inaba, "Full-body motion control integrated with force error detection for wheelchair support," IEEE-RAS International Conference on Humanoid Robots, pp. 193-198, 2011.

[6] S. Kajita and K. Tani, "Experimental study of biped dynamic walking in the linear inverted pendulum mode," Proceedings of 1995 IEEE International Conference on Robotics and Automation, vol. 3, pp. 28852891, 1995.

[7] L. Hawley and W. Suleiman, "External Force Observer for Mediumsized Humanoid Robots," in 2016 IEEE-RAS 16th International Conference on Humanoid Robots, 2016, pp. 366-371.

[8] S. Nozawa, R. Ueda, Y. Kakiuchi, K. Okada, and M. Inaba, "Sensorbased integration of full-body object manipulation based on strategy selection in a life-sized humanoid robot," Journal of Robotics and Mechatronics, vol. 23, no. 2, pp. 239-248, 2011.

[9] S. Kajita, F. Kanehiro, K. Kaneko, K. Fujiwara, K. Harada, K. Yokoi, and H. Hirukawa, "Biped Walking Pattern Generation by using Preview Control of Zero-Moment Point," in Proc. IEEE International Confer1626. 